\title{
Development of a Proximity and Tactile Sensor Array Using Self-Capacitance Measurement for Robot Hand
}

\author{
Satoshi Tsuji, ${ }^{\mathrm{a},}$, Teruhiko Kohama ${ }^{\mathrm{a}}$ \\ ${ }^{a}$ Dept. of Electrical Engineering, Fukuoka University, 8-19-1, Nanakuma, Jonan-ku, Fukuoka 814-0180, Japan \\ *Corresponding Author: tsuji@fukuoka-u.ac.jp
}

\begin{abstract}
We proposed a proximity and tactile sensor using self-capacitance measurement for robot hand. The proposed sensor consists of the two electrodes $\left(\mathrm{E}_{1}\right.$ and $\left.\mathrm{E}_{2}\right)$, GND and elastic body. The capacitance between $\mathrm{E}_{1}$ or $\mathrm{E}_{2}$ and GND is measured by switching between $\mathrm{E}_{1}$ and $\mathrm{E}_{2}$. The capacitance between $E_{1}$ and GND is used to detect the object before contact (proximity range) and to discriminate the material on contact. In addition, the capacitance between $\mathrm{E}_{2}$ and GND is used to detect the indentation on contact. In this paper, we propose the array system of this proposed sensor. In the experiment, detection of the object before and after contact, and identification of materials on contact were demonstrated. In addition, the prototype sensor array measured the shape of the object. The proposed sensor array may be useful as a tactile and proximity sensor on robot hand.
\end{abstract}

Keywords: Self-capacitance measurement, Tactile sensor, Proximity sensor, Robot hand.

\section{Introduction}

Nowadays, human-collaborative robots are rapidly emerging in our society. The robot should provide tactile sensor so that it carries out various tasks in collaboration with humans. So, many kinds of tactile sensor have already been developed ${ }^{(1)-(3)}$. Most of these sensors detect contact

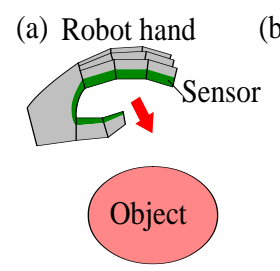

(b)

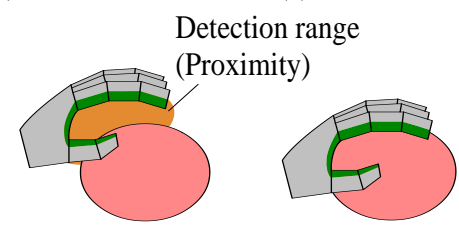

Proximity measurment Contact measurment

Fig. 1. The goal image of the proposed sensor. condition only. On the other hand, proximity sensors have been developed to detect the object before contact ${ }^{(4)}$. In addition, multiple tactile and proximity sensors have been proposed to obtain various information. Most of these sensors consist of some single sensing system. However, the wiring of the sensor may become complicated when covering a wide area. Previously, we proposed a new single unit tactile and proximity sensing method which simultaneously uses optical and electrical measurement to become the simple wiring ${ }^{(5)}$. However the sensor structure may become complicated because the proposed sensor consists of photodiode, LED and electrodes.

The goal of our research is to establish a simplified system of the tactile and proximity sensor for robot. Fig.1 shows the goal image that the sensor detects the object both before and after contact on the robot hand. When the robot hand approaches the object (Fig. 1(a)), the sensor detects the object and its position before contact (Fig. 1(b)). When the robot hand touches the object (Fig. 1(c)), the sensor detects the pressure and its position. Therefore, the sensor will assure the safety and workability of the robot because the robot will avoid unnecessary contact with objects including humans. We proposed a single unit tactile and proximity sensing method using self-capacitance measurement ${ }^{(6)}$. In this paper, we propose a proximity and tactile sensor array using self-capacitance measurement for robot hand. The array of the proposed sensor have been detected the object before and after contact. Therefore the structure becomes simple, and the sensor would improve the safety and workability of the robot.

\section{Principles}

In the proposed method, the self-capacitance measurement is used to detect the object both before and 


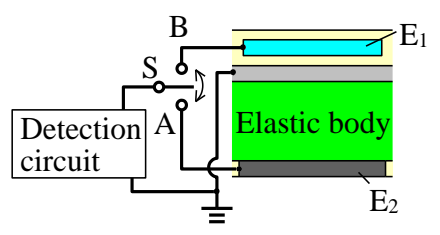

Fig. 2. Schematic diagram of the proposed method.
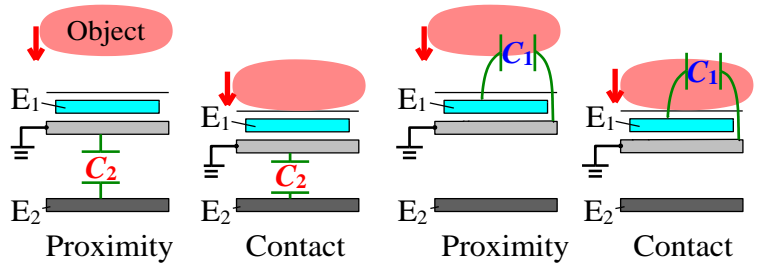

(a) Connection A

(b) Connection B

Fig. 3. The image of this measurement method.

after contact. Fig. 2 shows the schematic diagram of the proposed sensor. The proposed sensor consists of the two electrodes $\left(\mathrm{E}_{1}\right.$ and $\left.\mathrm{E}_{2}\right), \mathrm{GND}$ and elastic body. The capacitance between $E_{1}$ or $E_{2}$ and GND is measured by switching between $E_{1}$ and $E_{2}$. Fig. 3 shows the image for each measurement. As shown in Fig. 3(a), the capacitance $\left(C_{2}\right)$ between $\mathrm{E}_{2}$ and GND is measured to set the switch on A (Connection A). When the object approaches the sensor, $C_{2}$ does not change because GND are set between $\mathrm{E}_{2}$ and object. When the object touches the sensor, $C_{2}$ changes according to the indentation which is equal to the distance between $\mathrm{E}_{2}$ and GND. Here, the variation $\left(\Delta C_{2}\right)$ in $C_{2}$ is written by

$$
\Delta C_{2}=C_{2}-C_{20}
$$

Here, $C_{20}$ is steady-state value of $C_{2}$. Therefore, the sensor can detect the indentation (pressure) using $\Delta C_{2}$.

As shown in Fig. 3(b), the capacitance $\left(C_{1}\right)$ between $\mathrm{E}_{1}$ and GND is measured to set the switch on B (Connection B). When the object approaches the sensor, $C_{1}$ changes according to both the permittivity of the object and distance between the sensor and object. Here, $\Delta C_{1}$ is the variation in $C_{1}$. Therefore, the sensor can detect the object before contact when $\Delta C_{1}$ is changed at non-contact. In addition, the sensor can distinguish the material in the object from $\Delta C_{1}$ upon contact. Thus, the sensor can detect object both before and after contact using the self-capacitance measurement ${ }^{(6)}$.

\section{The Prototype Sensor and System}

Fig. 4 shows the structure of the prototype single sensor. The separation between the electrodes needs to be

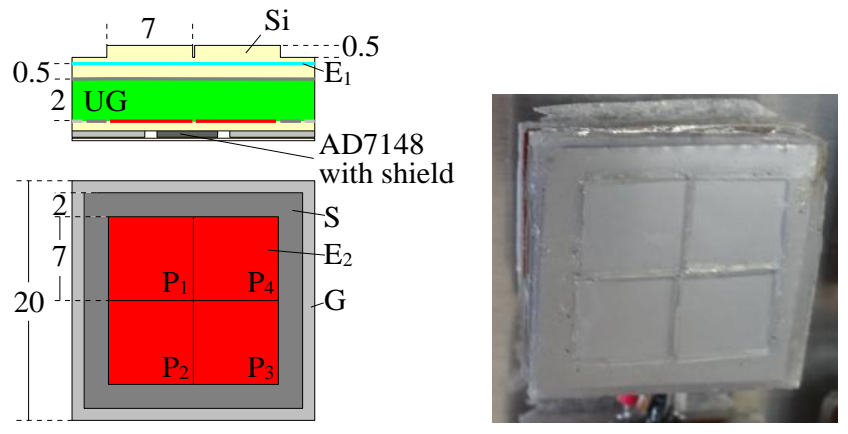

Fig. 4. Schematic diagram of the proposed sensor. $E_{1}$ and $\mathrm{E}_{2}$ : Electrode, UG: Urethane gel, Si: Silicone sheet, S: Shield, G: GND.

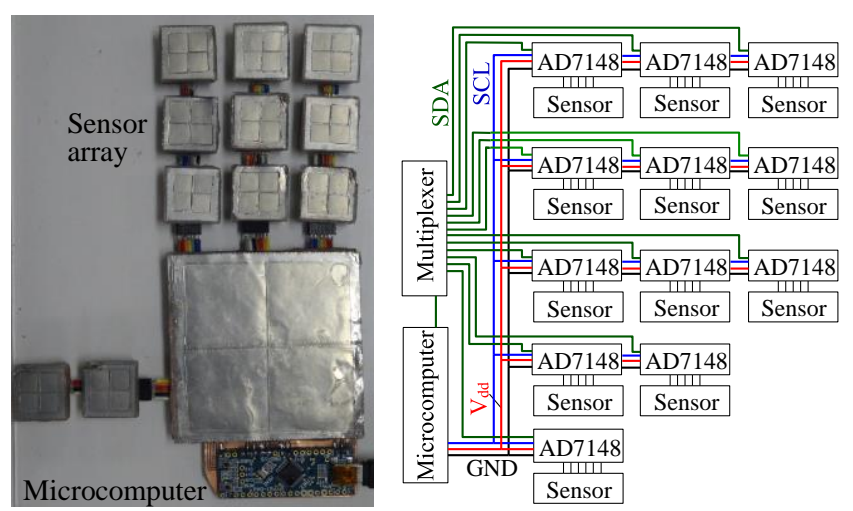

Fig. 5. Schematic diagram of the array system.

reduced to realize high spatial resolution. However, a large electrode is required to increase the sensitivity of electrical measurements. Thus, we propose a structure such that $E_{1}$ are large to increase the sensitivity at proximity range, and $E_{2}$ are small to realize a high spatial resolution on contact. Thus, in our design, one unit of the sensor consists of $2 \times 2$ electrodes for $E_{2}$, and one electrode for $E_{1}$. GND (G) electrode is set between $E_{1}$ and $E_{2}$. The urethane gel (thickness: $2 \mathrm{~mm}$, hardness: 5 (ASKER C), UG in Fig. 4) is set between $E_{1}$ and $E_{2}$. A silicone sheet (thickness: $0.1 \mathrm{~mm}$, $\mathrm{Si}$ in Fig. 4) is placed on top of the sensor that is isolated from the object. In addition, A silicone sheet (thickness: 0.5 $\mathrm{mm}, \mathrm{Si}$ in Fig. 4) is set on top of the sensor to increase the sensitivity of contact measurements. The capacitances are measured by capacitance measurement IC (Analog Devices, AD7148) which are capacitance-to-digital converter. AD7148 is attached to the back of the sensor to reduce electrical noise by wiring ${ }^{(6)}$.

Fig. 5 shows the structure of the prototype sensor array for robot hand. The sensor array consists of twelve sensors. The measured data (16 bit) by each AD7148 (slave device) are sent to a microcomputer (master device) by $\mathrm{I}^{2} \mathrm{C}$ serial interface bus. In addition, the measured data are sent to PC from a microcomputer by USB. $\mathrm{I}^{2} \mathrm{C}$ uses four linens which 
are serial data line (SDA), serial clock line (SCL), power line $\left(\mathrm{V}_{\mathrm{dd}}\right)$ and ground line $(\mathrm{GND})$. Each slave devices is connected to a master device by each serial data line and common lines which are serial clock, power and ground line. Thus, this array system needs the 15 wires which are twelve serial data lines, one serial clock line, one power line and one ground line. Each serial data lines are switched by the multiplexer continuously to send measurement data. Furthermore, the sensor can be increased on same $\mathrm{I}^{2} \mathrm{C}$ line. Thus, the wiring can be reduced. In addition, each AD7148 measure the capacitance of the sensor continuously when the other AD7148 send the data to microcomputer to reduce the response time.

\section{Experiment and Results}

\subsection{Proximity Measurement on Single Sensor}

We evaluated the proximity measurement using a sensor on the array system. The distance ( $d$ from 0 to 50 $\mathrm{mm}$ ) between the sensor and object was changed by the robot arm. The object and sensor were set up in the following conditions shown Fig. 6:

(A) The object covers the center on the sensor (Fig. 6(b)).

(B) The object covers a point $\left(\mathrm{P}_{1}\right)$ on the sensor (Fig. 6(c)).

The touch condition between the sensor and the object is detected from force gauge. The objects (The tip is a semicircle and the diameter is $20 \mathrm{~mm}$ ) are grounded conductors (GND) which are human models and acrylic.

Fig. 7 shows the variation $\left(\Delta C_{1}\right)$ which was measured by $\mathrm{E}_{1} . \Delta C_{1}$ changes according to the distance between the sensor and object. Here, when a priori information regarding the electrical property of the object is lacking, the sensor may not detect the distance between the sensor and object from $\Delta C_{1}$ because $\Delta C_{1}$ changes with both distance and the permittivity of the object. However, the sensor detected the approach of object and vice versa using $\Delta C_{1}$ before contact. In Fig. 7, the standard deviation (SD) of $\Delta C_{1}$ was 8.8 digits when the object was far from the sensor. Thus, the sensor can detect the object when $\Delta C_{1}$ is changed more than $\pm 3 \mathrm{SD}$ (26 digits) from a steady state value of $\Delta C_{1}$. In the case of the grounded conductor, the sensor detected the object within approximately $15 \mathrm{~mm}$, and in the case of the acrylic, the sensor detected the object within approximately $7 \mathrm{~mm}$.

Fig. 8 shows the variation $\left(\Delta C_{2}\right)$ which was measured by $\mathrm{E}_{2}$ on condition $\mathrm{A}$ of GND. In Fig. 8, $\Delta C_{2}$ does not change as the distance varies between $1 \mathrm{~mm}$ and $50 \mathrm{~mm}$

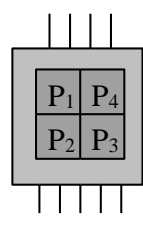

(a)

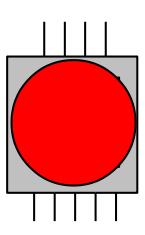

(b)

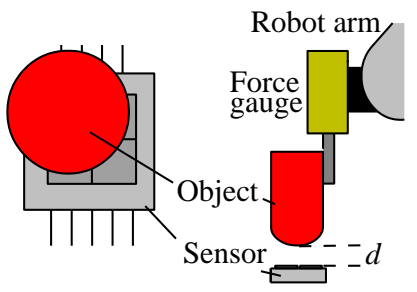

(c) (d)
Fig. 6. Measurement system. (a) Electrodes position. (b) Condition A. (c) Condition B. (d) Measurement position.

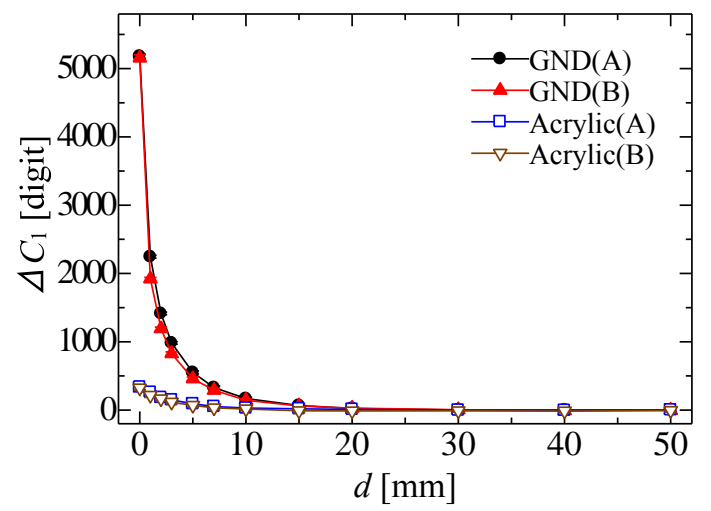

Fig. 7. Relationship between $\Delta C_{1}$ and $d$ at proximity range. Data are represented in mean $\pm \operatorname{SD}(n=15)$.

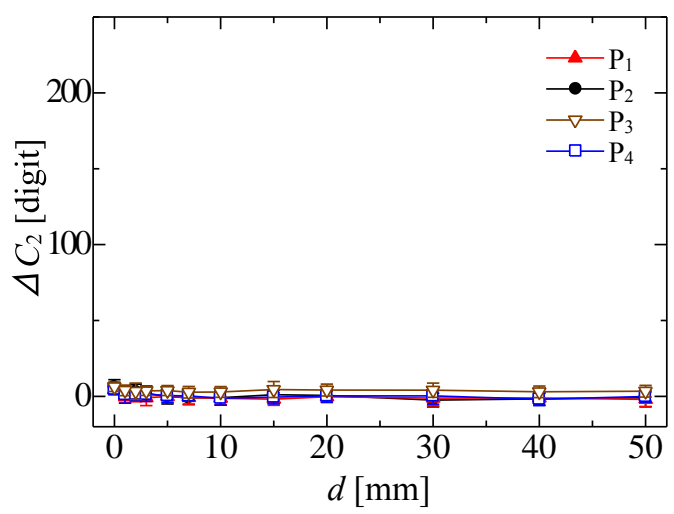

Fig. 8. Relationship between $\Delta C_{2}$ and $d$ at proximity range Data are represented in mean \pm SD $(n=15)$.

(proximity range). In addition, $\Delta C_{2}$ changes after contact. Thus, the sensor can detect the contact using $\Delta C_{2}{ }^{(6)}$. In Fig. 8 , the SD of $\Delta C_{2}$ was 2.5 digits when the object was far from the sensor. Thus, the sensor can detect the contact when $\Delta C_{2}$ is changed more than $\pm 3 \mathrm{SD}$ ( 7.5 digits) from a steady state value of $\Delta C_{2}$.

\subsection{Contact Measurement on Single Sensor}

We evaluated the contact measurement using a sensor on array system. The pressure (force) between the sensor and object is changed by robot arm. Force is measured by the force gauge. Fig. 9 shows the variation $\left(\Delta C_{1}\right)$ which was measured by $\mathrm{E}_{1}$. In Fig. $9, \Delta C_{1}$ changes with the permittivity of the object. The values of grounded 


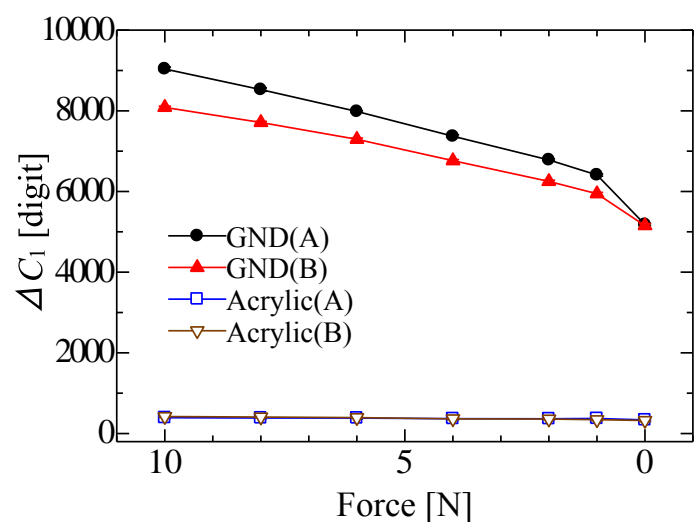

Fig. 9. Relationship between $\Delta C_{1}$ and force on contact. Data are represented in mean $\pm \mathrm{SD}(n=15)$.

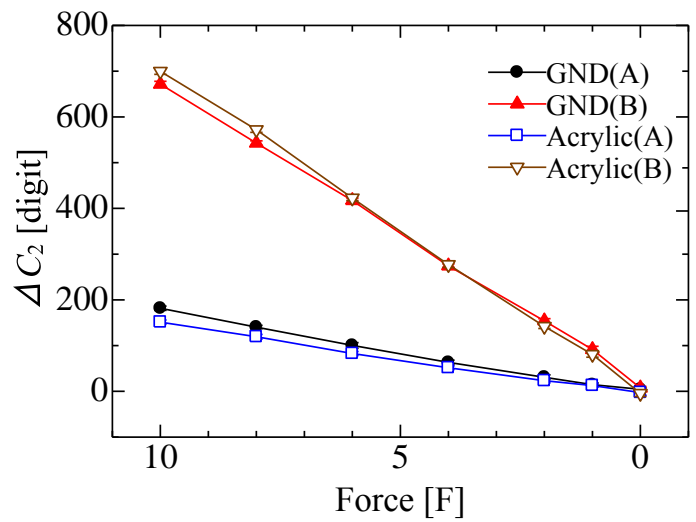

Fig. 10. Relationship between $\Delta C_{2}$ and force on contact. Data are represented in mean $\pm \mathrm{SD}(n=15)$.

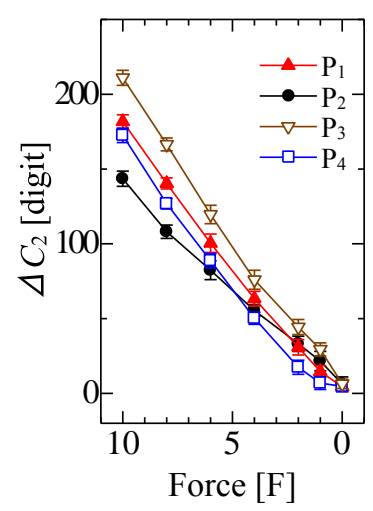

(a)

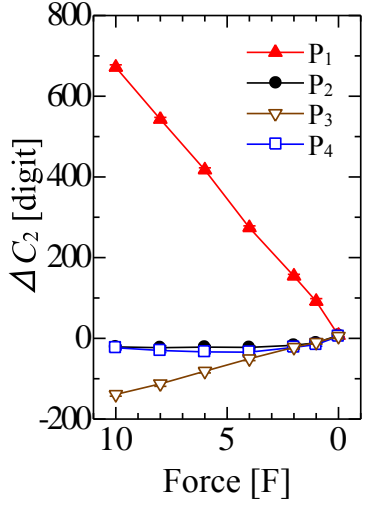

(b)
Fig. 11. Relationship between $\Delta C_{2}$ and force on contact. (a) Condition A. (b) Condition B. Data are represented in mean \pm SD $(n=15)$.

conductor are high, and the values of acrylic are low. Thus, the sensor can discriminate between the grounded conductor and other. Here, the conductance of human is high and a part of the human is grounded. Thus, the values of the finger are similarly changed as the grounded conductor. Therefore, the sensor can recognize whether the

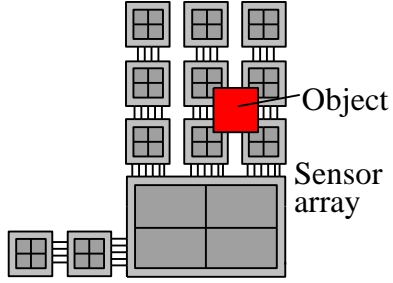

Fig. 12. The object position on the array system.
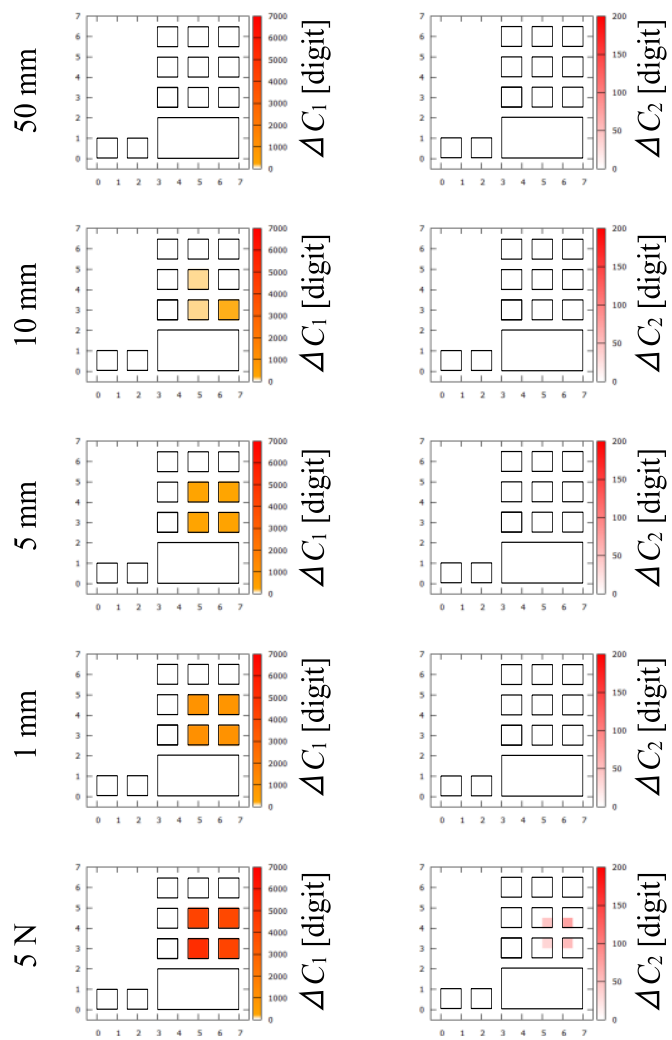

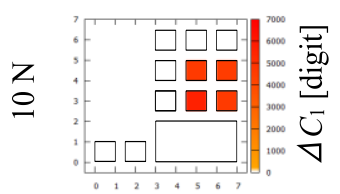

(a)

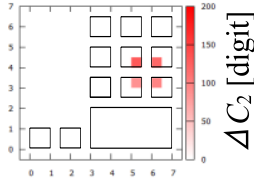

(b)
Fig. 13. Measured result of a GND object. (a) $\Delta C_{1}$. (b) $\Delta C_{2}$. object is a human (grounded conductor) or not ${ }^{(6)}$.

Fig. 10 shows $\Delta C_{2}$ for each experimental condition (A and B) on $\mathrm{P}_{1}$. Fig. 11(a) shows $\Delta C_{2}$ on condition $\mathrm{A}$ of the grounded conductor, and Fig. 11(b) shows $\Delta C_{2}$ on condition $\mathrm{B}$ of the grounded conductor. In Figs. 10 and 11, $\Delta C_{2}$ changes according to the force and position. In addition, $\Delta C_{2}$ does not change with the permittivity of the object. Therefore, the sensor can detect the pressure for each position $\left(\mathrm{P}_{1}-\mathrm{P}_{4}\right)$.

\subsection{The Array System of the Proposed Sensor}

We evaluated the measurement using all sensor of the 

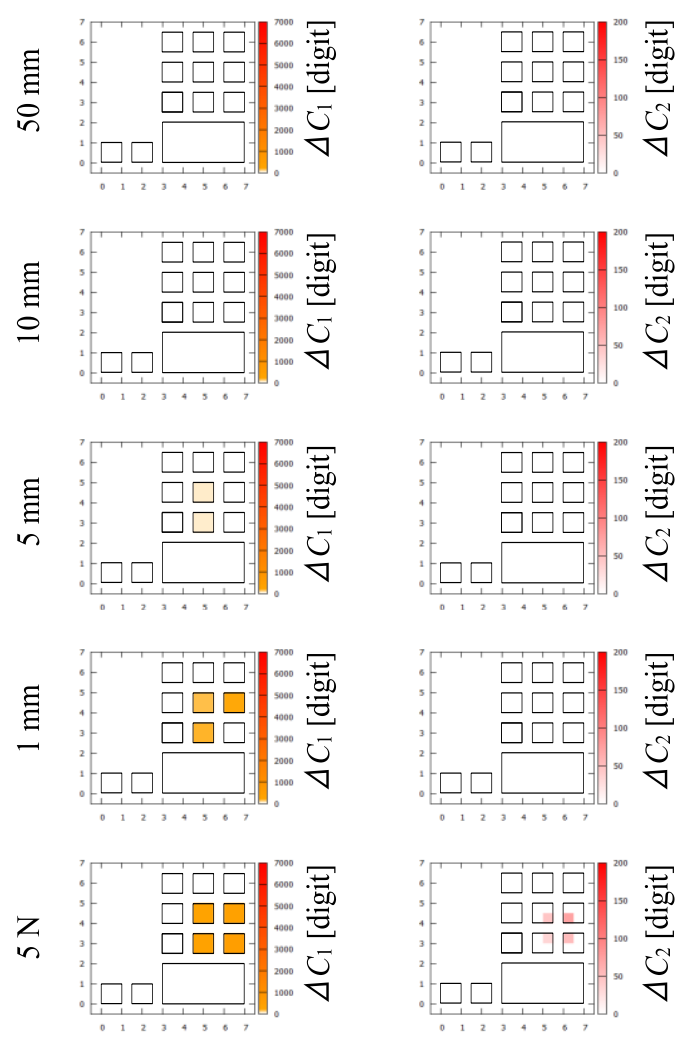

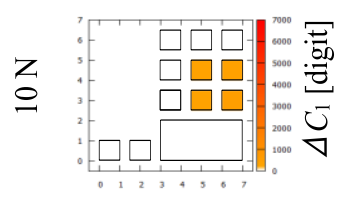

(a)

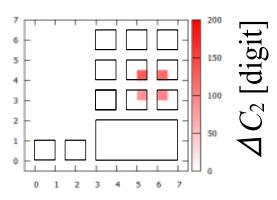

(b)
Fig. 14. Measured result of an acrylic object. (a) $\Delta C_{1}$. (b) $\Delta C_{2}$.

array system before and after contact. Fig. 12 shows the object position on the array system. Fig. 13 shows $\Delta C_{1}$ and $\Delta C_{2}$ when the object is a grounded conductor (GND, $20 \times$ $20 \mathrm{~mm}$ ), and Fig. 14 shows $\Delta C_{1}$ and $\Delta C_{2}$ when the object is an acrylic object $(20 \times 20 \mathrm{~mm})$. Figs. 13(a) and 14(a) show $\Delta C_{1}$, and Figs. 13(b) and 14(b) show $\Delta C_{2}$. Here, $\Delta C_{1}$ and $\Delta C_{2}$ became the white color in Figs. 13 and 14 when the values are less than \pm 3 SD lower than each value of air. In Figs. 13(a) and 14(a), $\Delta C_{1}$ change according to the distance and the permittivity of the object at non-contact. Thus, the sensor can detect the object and position before contact (proximity range). In addition, the sensor can discriminate between a grounded conductor and another after on contact.

In Figs. 13(b) and 14(b), $\Delta C_{2}$ changes according to the pressure and the precise position of the object when the object is pressed. In addition, $\Delta C_{2}$ does not change without contact. Thus, the sensor can detect the pressure and the precise position of the object. Therefore, the sensor would improve the safety and workability of the robot. It takes approximately $64 \mathrm{~ms}$ to obtain one complete cycle of measurements (12 sensors) with the data transmitting time from a microcomputer to PC. This data transmitting time from a microcomputer to $\mathrm{PC}$ is approximately $46 \mathrm{~ms}$. Thus, the main time-consuming of this system are the data transmitting time. Therefore, we think that the response time of this system can be reduced to reduce the data transmitting time.

\section{Conclusions}

In this study, we propose a proximity and tactile sensor array using self-capacitance measurement for robot hand. The proposed sensor consists of the two electrodes $\left(E_{1}\right.$ and $\mathrm{E}_{2}$ ), GND and elastic body. In the experiment, detection of the object before and after contact, and identification of materials on contact were demonstrated. In addition, the prototype sensor array measured the shape of the object. The proposed sensor array may be useful as a tactile and proximity sensor on robot hand.

\section{References}

(1) K. Kamiyama, K. Vlack, T. Mizota, H. Kajimoto, N. Kawakami, and S Tachi : "Vision-Based Sensor for Real-Time Measuring of Surface Traction Fields", IEEE Computer Graphics and Applications, Vol.25, No.1, pp.68-75, 2005

(2) T. Hoshi, and H. Shinoda : "Robot Skin Based on Touch-Area-Sensitive Tactile Element”, Proc.2006 IEEE International Conference on Robotics and Automation, pp.3463-3468, 2006

(3) M. Shimojo, T. Araki, A. Ming, and M. Ishikawa : “A High-Speed Mesh of Tactile Sensors Fitting Arbitrary Surfaces", IEEE Sensor Journal, Vol.10, No.4, pp.822-830, 2010

(4) K. Hsiao, P. Nangeroni, M. Huber, A. Saxena and A.Y. $\mathrm{Ng}$ : "Reactive Grasping Using Optical Proximity Sensors", Proc.2009 IEEE International Conference on Robotics and Automation, pp.2098-2105, 2009

(5) S. Tsuji, and T. Kohama : "An Array System of Proximity and Tactile Sensors by Simultaneous Measurement of Optical and Electrical Properties", IEEJ Trans. SM, Vol.133, No.3, pp.66-71, 2013

(6) S. Tsuji, and T. Kohama : "A Proximity and Tactile Sensor Using Self-Capacitance Measurement", IEEJ Trans. SM, Vol.134, No.12, pp.400-405, 2014 (in Japanese) 